TRANSACTIONS OF THE

AMERICAN MATHEMATICAL SOCIETY

Volume 350, Number 7, July 1998, Pages 2779-2796

S 0002-9947(98)02182-5

\title{
ON THE MEASURE THEORETIC STRUCTURE OF COMPACT GROUPS
}

\author{
S. GREKAS AND S. MERCOURAKIS
}

\begin{abstract}
If $G$ is a compact group with $w(G)=a \geq \omega$, we show the following results:

(i) There exist direct products $\prod_{\xi<a} G_{\xi}, \prod_{\xi<a} H_{\xi}$ of compact metric groups and continuous open surjections $\prod_{\xi<a} G_{\xi} \stackrel{p}{\rightarrow} G \stackrel{q}{\rightarrow} \prod_{\xi<a} H_{\xi}$ with respect to Haar measure; and

(ii) the Haar measure on $G$ is Baire and at the same time Jordan isomorphic to the Haar measure on a direct product of compact Lie groups.

Applications of the above results in measure theory are given.
\end{abstract}

\section{INTRODUCTION}

Our aim in this note is the investigation of the measure theoretic structure of a compact group. We have been motivated by the following classical results of Kuzminov [Kuz], every compact group $G$ is a dyadic space (i.e., a continuous image of a space of the form $\{0,1\}^{a}$ ) and Sapirovskii, [Co-Ho-Re], every compact group $G$ admits a continuous mapping onto the cube $[0,1]^{a}$, where $a=w(G)$. Both of these results are purely topological and do not involve the group structure of $G$.

In the first section of this note we show (after replacing the spaces $\{0,1\}^{a}$ and $[0,1]^{a}$ by proper direct products of compact metric groups) that we can choose the continuous surjections of both of the above theorems to be open and to respect Haar measure (Theorems 1.1 and 1.4). In fact we show that in a certain sense they are very close to being continuous epimorphisms (see Remarks 1.2, 1.5 and Corollaries 1.3, 1.6).

M.M. Choban has proved that every compact group is Baire isomorphic to a direct product of compact metric groups [Ch]. In a recent paper, the first named author proved that this Baire isomorphism can be constructed so that it takes Haar measure to Haar measure $\left[\mathrm{G}_{2}\right]$. In the second section of this paper we deal with an improvement of this result. We show that this isomorphism has the further property to be a "Jordan" isomorphism (see Def. 2.6 and Theorem 2.13). This particularly implies that the space of Riemann integrable functions on a compact group is isometric to the space of Riemann integrable functions on a product of compact metric groups (Corollary 2.15).

Received by the editors February 1, 1996.

1991 Mathematics Subject Classification. Primary 22C05, 28A35; Secondary 43A05.

Key words and phrases. Compact group, Haar measure, Baire isomorphism, Riemann integrable function, Jordan measurable set. 
Several applications of the above results are given. Among these we mention Theorem 1.10 (a direct proof of the existence of a strong lifting on a compact group) and Theorem 2.17 (a rather illuminating proof of the existence of a uniformly distributed sequence on a compact separable group).

Preliminaries and notation. We shall denote by $\omega(=\{1,2, \ldots\})$ the (cardinality of the) set of natural numbers, by $\omega^{+}$the first uncountable cardinal, and by $c$ the cardinality of the continuum. If $S$ is any set we denote by $|S|$ its cardinality. By a compact space (resp. compact group) we mean a compact Hausdorff topological space (resp. compact Hausdorff topological group). If $X$ is any (compact) space then by $w(X)$ we denote the topological weight of $X$. By the term Haar measure on the compact group $G$ we mean the normalized Haar measure (usually denoted by $\lambda_{G}$ ) on $G$.

In the present paper we shall repeatedly use the following classical results concerning compact groups.

Theorem (A) (Pontrjagin-van Kampen, [Pr, Th. 6.5.6]). Let G be a (non-trivial) compact connected group and $A$ the connected component of the center of $G$. Then there exist a family $\left\{H_{i}: i \in I\right\}$ of compact simply connected simple Lie groups, each with a finite center $C_{i}$, and a totally disconnected closed subgroup $N$ of the center of $A \times \prod_{i \in I} H_{i}$ such that $G$ is topologically isomorphic to the quotient $A \times \prod_{i \in I} H_{i} / N$.

So if we set $H=\prod_{i \in I} H_{i}$ and $C=\prod_{i \in I} C_{i}$, then (the center of $A \times H$ is the group $A \times C$ and) clearly we have the following continuous epimorphisms: $A \times H \stackrel{\phi}{\rightarrow} G \stackrel{\psi}{\rightarrow}$ $H / C$, where $\phi$ has as a kernel the group $N$ and $\psi$ the group $\phi(A \times C)$. Using the fact that $\left|C_{i}\right|<\omega$ for each $i \in I$, we get that $w\left(H_{i}\right)=\omega$ for all $i \in I$; since $A$ is a topological subgroup of $G$, we conclude that $w(G)=\max \{w(A), \omega,|I|\}$ (cf. the proof of Th. 2.4 of [Cl-Mo2] and the proof of Th. 6.5 of [Co-Re]).

Theorem (B) (Mostert, [Mos, Th. 8]). Let $G$ be a compact group and $G_{0}$ the connected component of $G$. Then $G$ is homeomorphic to $G / G_{0} \times G_{0}$.

Some further remarks concerning this result are stated below. P. Mostert has in fact proved that if $G$ is a compact group and $G_{0}$ is a closed subgroup of $G$ such that the quotient $G / G_{0}$ is a totally disconnected space, then there exists a continuous mapping (a full cross section) $f: G / G_{0} \rightarrow G$ such that $\pi(f(\dot{x}))=\dot{x}$ for each $\dot{x} \in G / G_{0}$, where $\pi: G \rightarrow G / G_{0}: x \rightarrow \dot{x}$ is the canonical map. It then easily follows that the map $\Phi: G / G_{0} \times G_{0} \rightarrow G$ defined by the rule $\Phi(t, \dot{x})=f(\dot{x}) \cdot t$, is a homeomorphism between $G / G_{0} \times G_{0}$ and $G$. If $G_{0}$ is in addition a normal subgroup of $G$ and we denote by $\lambda, a, \beta$ the Haar measure on $G, G / G_{0}, G_{0}$, respectively, then it can be proved by using standard results that $\Phi(a \otimes \beta)=\lambda$ (see for instance [D], $14.4 .1,14.4 .2$, p. 266 and also $\left[\mathrm{G}_{1}\right]$, Remarks 3.5.1). In the case when $G_{0}$ is the connected component of $G$, it is well known that the quotient group $G / G_{0}$ is totally disconnected and hence we get Theorem (B) (with the further property concerning Haar measure).

If $X$ is any compact space we denote by $P(X)$ the set of Radon probability measures on $X$. A Radon measure $\mu$ on $X$ is said to be completion regular if for every Borel subset $A$ of $X$ there exist Baire sets $B, \Gamma$ such that $B \subseteq A \subseteq \Gamma$ and $\mu(\Gamma \backslash B)=0$. It is well known that the Haar measure on a compact group (and 
also any strictly positive product measure on a product of compact metric spaces) is completion regular. Let $\mu \in P(X)$. A bounded function $f: X \rightarrow \mathbb{R}$ is said to be $\mu$-Riemann integrable if the set of points of discontinuity of $f$ has $\mu$-measure zero. It follows from Lusin's theorem that each $\mu$-Riemann integrable function is $\mu-$ measurable (and thus $\mu$-integrable). A subset $A$ of $X$ is called $\mu$-Jordan measurable if its characteristic function $\mathcal{X}_{A}$ is $\mu$-Riemann integrable. It is well known that: (i) The set $J_{\mu}(X)$ of $\mu$-Jordan measurable sets is a Boolean algebra with the usual set-theoretic operations (called the algerba of $\mu$-Jordan measurable sets), and (ii) the set of open (resp. open and Baire) members of $J_{\mu}(X)$ is a basis for the topology

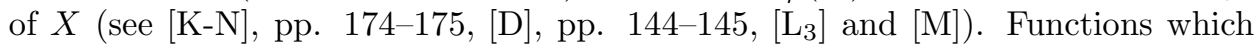
are $\mu$-equivalent to Riemann integrable functions are characterized as follows:

Theorem (C) (see [D, 13.9, ex. 6, p. 144] and $\left[\mathrm{L}_{3}\right]$ ). Let $X$ be a compact space, $\mu \in P(X)$ a strictly positive measure (i.e. $\mu(U)>0$ for every open non-empty subset $U$ of $X)$ and $f: X \rightarrow \mathbb{R}$ a bounded function. Then the following are equivalent:

(i) There is a $\mu$-Riemann integrable function $g$ such that $f=g \mu-a . e$.

(ii) There is $A \subseteq X$ with $\mu(A)=1$ such that the function $f \mid A$ is (relatively) continuous on $A$.

(iii) The function $f$ is $\mu$-equivalent both to an upper semicontinuous and to a lower semicontinuous function.

Let $\mu \in P(X)$ be a strictly positive measure. We denote by $J_{\mu}$ the quotient algebra $J_{\mu}(X)$ modulo sets of $\mu$-measure zero and call it the Jordan algebra of $\mu$. It follows from Theorem (C) that $J_{\mu}$ can be identified with the subalgebra of the measure algebra $\mathcal{B}_{\mu}$ of $\mu$, consisting of those classes each containing an open and a closed set simultaneously (see $\left[\mathrm{L}_{3}\right]$ and $\left.[\mathrm{M}]\right)$. Let $X\left(J_{\mu}\right)$ be the Stone space of $J_{\mu}$ and $\bar{\mu}$ the induced probability measure on $\left(J_{\mu}\right.$ and hence on) $X\left(J_{\mu}\right)$. It then follows that a continuous and onto mapping $\pi: X\left(J_{\mu}\right) \rightarrow X$ can be defined such that $\pi(\bar{\mu})=\mu$. The mapping $\pi$ has the following properties: (i) If $A \in J_{\mu}(X)$, then $\pi(\widehat{A})=A^{0-}$ and (ii) if $F \subseteq X\left(J_{\mu}\right)$ is compact, then $\bar{\mu}(F)=\mu(\pi(F))$ (see $\left[\mathrm{L}_{3}\right]$, Lemma 1 and 2 , and also $[\mathrm{M}])$.

Let $X, Y$ be compact spaces, $\mu \in P(X), \nu \in P(Y)$ and $\mathcal{B}_{1}, \mathcal{B}_{2}$ Boolean subalgeras of the measure algebras $\mathcal{B}_{\mu}, \mathcal{B}_{\nu}$ respectively. We say that the algebra $\mathcal{B}_{1}$ is embedded in the algebra $\mathcal{B}_{2}$ (resp. $\mathcal{B}_{1}$ and $\mathcal{B}_{2}$ are isomorphic) if there exists a one-to-one (resp. one-to-one and onto) map $\phi: \mathcal{B}_{1} \rightarrow \mathcal{B}_{2}$, with $\phi(\widehat{A} \cup \widehat{B})=\phi(\widehat{A}) \cup \phi(\widehat{B})$, $\phi(\widehat{X} \backslash \widehat{A})=\widehat{Y} \backslash \phi(\widehat{A})$ and $\nu(\phi(\widehat{A}))=\mu(\widehat{A})$ for $\widehat{A}, \widehat{B} \in \mathcal{B}_{1}$.

1.

The first of our main results extends and refines a classical result of Kuzminov [Kuz]: Every compact group is a dyadic space, i.e. a continuous image of a space of the form $\{0,1\}^{a}$, where $a=w(G)$ (see also, [H-R], p. 106, [Co], [Co-Ho-Re], pp. 93-94 and the references given there). Theorems (A) and (B) stated in the preliminaries will be crucial in the proof of our result as they were in the proof of a similar result of Pelczynski (see Prop. 7.6 of [P] and also [Cl-Mo 1 , [Cl-Mo2]).

We think that the following terminology is convenient for our purposes: Two compact groups $G_{1}$ and $G_{2}$ will be called Haar homeomorphic, if there exists a homeomorphism between of them (called a Haar homeomorphism) that takes Haar measure to Haar measure. It is clear that if $G_{1}, G_{2}$, are topologically isomorphic 
then they are Haar homeomorphic. In this terminology Theorem (B) (Mostert's theorem) says that, if $G$ is any compact group then $G$ is Haar homeomorphic to the group $G / G_{0} \times G_{0}$.

Theorem 1.1. Let $G$ be a compact group such that $w(G)=a \geq \omega$. Then there exists a family $G_{\xi}, \xi<a$, of compact metric groups (each having at least two points if $\left.a \geq \omega^{+}\right)$and a continuous open and onto map $f: \prod_{\xi<a} G_{\xi} \rightarrow G$ such that $f\left(\bigotimes_{\xi<a} \lambda_{\xi}\right)=\lambda$, where $\lambda, \lambda_{\xi}$ denote the Haar measure on $G, G_{\xi}$ respectively. Furthermore:

(1) If $G$ is connected or abelian, the map $f$ can be chosen to be a homomorphism;

(2) If $G$ is totally disconnected, the map $f$ can be chosen to be a homeomorphism and each of the groups $G_{\xi}$ finite.

Proof. We first notice that Theorem (B) (and the remarks following it) imply that it is enough to show the theorem in cases (1) and (2) separately. Indeed, let's assume for the moment that cases (1) and (2) of the above theorem have been proved, and let $G$ be an arbitrary compact group. It then follows from Theorem (B), that $G$ is Haar homeomorphic to the group $G / G_{0} \times G_{0}$; since the group $G / G_{0}$ is totally disconnected there is, from (2) a direct product of compact metric groups $\prod_{i \in I} H_{i}$ that is Haar homeomorphic to $G / G_{0}$. It follows immediately that $G$ is Haar homeomorphic to the group $\prod_{i \in I} H_{i} \times G_{0}$. Now it follows from (1) that there is a direct product of compact metric groups $\prod_{j \in J} F_{j}$ and a continuous homomorphism $\pi_{1}$ from $\prod_{j \in J} F_{j}$ onto $G_{0}$. It is clear that the map $\pi=i d \otimes \pi_{1}: \prod_{i \in I} H_{i} \times \prod_{j \in J} F_{j} \rightarrow \prod_{i \in I} H_{i} \times G_{0}$, where $i d$ denotes the identity map on $\prod_{i \in I} H_{i}$, is a continuous epimorphism. This finishes the proof of our assertion.

Before we proceed to the proof of the theoren we should notice that: If $G$ is any compact abelian group (of weight $a \geq \omega$ ) then it is well known that it is topologically isomorphic to a quotient of a direct product $\prod_{\xi<a} G_{\xi}$ of compact metric (abelian) groups; this result is due to Vilenkin (see [H-R], 25.35, p. 423). If $G$ is a compact totally disconnected group then it is also well known that $G$ is homeomorphic to the space $\{0,1\}^{a}$, where $a=w(G) \geq \omega$; this result is due to Ivanovskii (see [H-R], Th. 9.15, p. 95). Therefore case (2) of our result improves the result of Ivanovskii.

(1) Assume that $\mathbf{G}$ is connected. By Theorem (A) there is a family $\left\{H_{i}: i \in I\right\}$ of compact (simply connected, simple) Lie groups such that $G$ is a quotient of the product $A \times \prod_{i \in I} H_{i}$, where $A$ is the connected component of the center of $G$; since $A$ is a compact abelian group, the above mentioned result of Vilenkin (and the equality $w(G)=\max (w(A), \omega,|I|)$ of the preliminaries) finishes the proof of this case.

(2) Assume that $G$ is totally disconnected. In $\left[\mathrm{G}_{2}\right]$, Th. 2.3, it is shown that the Haar measure on any compact group is Baire isomorpic to the Haar measure on 
some direct product of compact Lie groups (see also section 2 of the present paper for an extension of this result). We adapt that proof in order to achieve (whenever $G$ is totally disconnected) a homeomorphism with the same property.

Claim (I) (cf. Lemma 2.1 of $\left[\mathrm{G}_{2}\right]$ and Lemma 2.11 of section 2 infra). If $H$ is a finite normal subgroup of $G$, then there is a compact and open set $S \subseteq G$ such that:

(i) $G=S \cdot H$,

(ii) the mapping $\left.\pi\right|_{s}$ is one-to-one and onto $G / H$, where $\pi: G \rightarrow G / H$ is the canonical map and (hence)

(iii) the bijection $q: G / H \times H \rightarrow G$ defined by $q(\dot{x}, t)=r(\dot{x}) \cdot t$, where, $r(\dot{x})=$ $\pi^{-1}(\dot{x}) \cap S$, is a homeomorphism with $q(a \otimes \beta)=\mu$ (where $\mu, a, \beta$ denote the normalized Haar measure on $G, G / H, H$ respectively).

Proof of Claim (I). It is an immediate consequence of the results of Mostert mentioned in the preliminaries. Despite this fact we prefer to give a direct (simple) proof of Claim (I). Since $G$ is a compact totally disconnected group the set of normal open subgroups of $G$ is a neighbourhood basis for the identity $e$ in $G$ (see Th. 7.7 in [H-R]). So let $F$ be an open subgroup of $G$ with $F \cap H=\{e\}$ (recall that $H$ is finite). We notice that $F$ is compact (because each open subgroup of a topological group is also closed), and also that (as it is readily verified) the map $\left.\pi\right|_{F}$ is one-to-one. Therefore $\pi(F)=\pi(F \cdot H)$ is a compact and open subgroup of $G / H$, topologically isomorphic to $F$. So if $x, y \in G$, then the sets $\pi(x F), \pi(y F)$ either are disjoint or coincide. Now it easily follows from compactness that there are $y_{1}, \ldots, y_{n} \in G$ such that the mapping $\pi$ restricted to the set $S=\bigcup_{i=1}^{n} y_{i} \cdot F$ is one-to-one and onto $G / H$. It is plain that conditions (i), (ii) and (iii) are satisfied by the triplet $(S, q, r)$.

Claim (II) (cf. Lemma 2.2 of $\left[\mathrm{G}_{2}\right]$ and Lemma 2.12 of section 2 infra). There is a family $\left(H_{\xi}\right)_{\xi \leq a}$ of closed normal subgroups of $G$ with $\bigcap_{\xi<a} H_{\xi}=\{e\}$ and $H_{0}=G$ such that:

(i) $H_{\xi} \subseteq H_{\zeta}$ for $0 \leq \zeta<\xi \leq a$,

(ii) $H_{\xi} / H_{\xi+1}$ is finite for each $\xi<a$, and

(iii) $H_{\xi}=\bigcup_{\zeta<\xi} H_{\zeta}$ for each limit ordinal $\xi \leq a$.

Proof of Claim (II). We consider the set $\mathcal{B}$ of open (and hence compact) normal subgroups of $G$ (we recall that $\mathcal{B}$ is a neighbourhood basis for the identity of $G$ ). Let $\mathcal{B}=\left\{F_{\xi}: \xi<a\right\}$ be an enumeration of $\mathcal{B}$. We set $H_{0}=F_{0}(=G), H_{\xi}=H_{\zeta} \cap F_{\xi}$ if $\xi=\zeta+1$ for some $\zeta<a$, and $H_{\xi}=\bigcap_{\zeta<\xi} H_{\zeta}$ for each limit ordinal $\xi \leq a$. It is then easily proved that the family $\left\{H_{\xi}: \xi \leq a\right\}$ has the properties (i) to (iii).

In order to finish the proof of (2), set $G_{0}=G / H_{1}$ and $G_{\xi}=H_{\xi} / H_{\xi+1}$ for $0<\xi<$ $a$, (hence each $G_{\xi}$ is a finite group) and denote by $\lambda_{\xi}$ the (normalized) Haar measure on $G_{\xi}$. By using induction on $\xi<a$, standard arguments involving projective limits of compact spaces and Claims (I) and (II), we can find a homeomorphism $f$ from the compact group $\prod_{\xi<a} G_{\xi}$ onto $G$ such that $f\left(\otimes_{\xi<a} \lambda_{\xi}\right)=\lambda$. We omit the details of this proof and instead we refer the reader to $\left[\mathrm{G}_{2}\right]$ (Th. 2.3). 
The proof of the theorem is complete.

Remark 1.2. (1) We should notice that a restatement of Theorem 1.1 goes as follows: If $G$ is any compact group with $w(G)=a \geq \omega$, then there exists a compact group $G^{\prime}$ Haar homeomorphic to $G$, a direct product $\prod_{\xi<a} G_{\xi}$ of compact metric groups and a continuous epimorphism $f: \prod_{\xi<a} G_{\xi} \rightarrow G^{\prime}$ such that, if $G$ is connected or abelian then $G$ is topologically isomorphic to $G^{\prime}$, and if $G$ is totally disconnected then $f$ is the identity map and each $G_{\xi}$, finite.

(2) The property $f\left(\bigotimes_{\xi<a} \lambda_{\xi}\right)=\lambda$ of the map $f$ of Theorem 1.1 can be put in a more general setting: If $\nu$ is any completion regular measure on $G$ (not necessarily the Haar measure) then there exists a completion regular measure $\mu$ on $\prod_{\xi<a} G_{\xi}$ such that $f(\mu)=\nu$. This fact is an immediate consequence of Theorem 1.1 (or its equivalent form stated in Remark $1.2(1)$ above) and of Prop. 3.1 in $\left[\mathrm{G}_{3}\right]$ which says that: If $\pi$ is any continuous homomorphism from the compact group $G_{1}$ onto the compact group $G_{2}$ then for every completion regular measure $\mu_{2}$ on $G_{2}$ there is a completion regular measure $\mu_{1}$ on $G_{1}$ such that $\pi\left(\mu_{1}\right)=\mu_{2}$.

An obvious consequence of Remark 1.2 (1) is the following improvement of the above mentioned result of Pelczynski (Prop. 7.6 of $[\mathrm{P}]$ ).

Corollary 1.3. Every compact group is Haar homeomorphic to a quotient group of a direct product of a family of compact metric groups (and thus a dyadic space).

The second of the main results of this section is, in a sense, the dual of Theorem 1.1.

Theorem 1.4. Let $G$ be a compact group such that $w(G)=a \geq \omega$. Then there exist a family $G_{\xi}, \xi<$ a of compact metric (Lie) groups (each having at least two points if $\left.a \geq \omega^{+}\right)$and a continuous open and onto map $f: G \rightarrow \prod_{\xi<a} G_{\xi}$ such that $f(\lambda)=\bigotimes_{\xi<a} \lambda_{\xi}$, where $\lambda, \lambda_{\xi}$ is the Haar measure on $G, G_{\xi}$ respectively. Furthermore:

(1) If $G$ is connected or abelian the map $f$ can be chosen to be a homomorphism, and (by Case (2) of Theorem 1.1)

(2) if $G$ is totally disconnected the map $f$ can be chosen to be a homeomorphism and each of the groups $G_{\xi}$ finite.

Proof. We first recall the classical result that, if $G$ is any compact group and $U$ is a neighbourhood of the identity in $G$ then there is a closed normal subgroup $H$ of $G$ with $H \subseteq U$, such that $G / H$ is a Lie group (see [Mon-Zip] and also [Pr], pp. 140-141). It clearly follows that we may restrict our attention to the case $a \geq \omega^{+}$.

Using Mostert's result (Theorem (B)) once again the proof is clearly reduced to cases (1) and (2) separately. The second case has already been proved in Theorem 1.1, so it remains to examine the first case. Assume firstly that $G$ is abelian (connected or not). Then, since $w(G)=w(\widehat{G})=|\widehat{G}|=a \geq \omega^{+}$(where $\widehat{G}$ denotes the dual group of $G$ which is of course a discrete abelian group), we get that the $\operatorname{rank} r(\widehat{G})$ of $\widehat{G}$ is equal to $|\widehat{G}|$ (see Th. A.14, A.15, A.16 of $[\mathrm{H}-\mathrm{R}]$ or Th. 2.1 of $[\mathrm{Co}-\mathrm{Re}])$. So there exists an algebraically independent set $X$ of characters 
of $G$ with $|X|=|\widehat{G}|=a$. It then follows from a result of D.A. Edwards [E] that ( $X$ is a stochastically independent set of random variables for the probability space $(G, \lambda)$ and hence) the mapping $\phi: G \rightarrow \prod_{x \in X} K_{x}: g \rightarrow(x(g))_{x \in X}$, where $K_{x}=x(G) \subseteq T=\left\{e^{2 \pi i t}: t \in[0,1]\right\}$ for $x \in X$, is a continuous epimorphism (see also [Co-Re], Lemma 5.4). So we have proved the result for abelian $G$.

If we assume that $G$ is connected, then from Theorem (A) we get the following continuous epimorphisms, $A \times H \stackrel{\phi}{\rightarrow} G \stackrel{\psi}{\rightarrow} H / G \cong \prod_{i \in I}\left(H_{i} / C_{i}\right)$ (see the preliminaries).

As we have $w(G)=\max \{w(A), \omega,|I|\}$, we distinguish two cases for $w(G)=a$ : either $w(G)=w(A)$ or $w(G)=|I|$.

(A). Assume that $w(G)=w(A)$. In this case we follow Comfort and Remus in order to show the result (cf. the proof of Th. 6.5 of [Co-Re]). It is easy to see that there is a closed totally disconnected subgroup $B$ of $A$ such that $N \subseteq B \times C$, where $N$ is the kernel of $\phi$. We then have the following chain of continuous epimorphisms,

$$
A \times H \rightarrow G \rightarrow(A \times H) /(B \times C) \cong(A / B) \times(H / C) \rightarrow A / B
$$

Since $B$ is a closed totally disconnected subgroup of the compact connected abelian group $A$, we get $w(A)=w(A / B)$ according to Lemma 6.4 of [Co-Re], and since the group $A / B$ is abelian, we get, from the above and $(*)$, the desired conclusion.

(B). Assume that $w(G)=|I|$. Then clearly we can take the epimorphism $\psi$ as the desired map $f$.

The proof of the theorem is complete.

Remark 1.5. (1) It is easily seen that observations similar to those mentioned in Remark 1.2 hold true also for Theorem 1.4. So we have for instance: If $G$ is any compact group with $w(G)=a \geq \omega^{+}$, then there exists a compact group $H$, Haar homeomorphic to $G$, a direct product $\prod_{\xi<a} G_{\xi}$ of compact metric groups (each $G_{\xi}$ having at least two points) and a continuous epimorphism $f: H \rightarrow \prod_{\xi<a} G_{\xi}$ such that, if $G$ is connected or abelian then $G$ is topologically isomorphic to $H$, and if $G$ is totally disconnected then $f$ is the identity mapping and each $G_{\xi}$, finite.

(2) We notice that Theorem 1.4 can be considered as a refinement of the following well known result of Sapirovskii: If $G$ is a compact group of weight $a \geq \omega$, then there is a continuous map $f$ from $G$ onto the cube $[0,1]^{a}$ (see [Co-Ho-Re], pp. 83, and [Cl-Mo2]).

An immediate consequence of Theorem 1.4 (either of Remark 1.5 (1)) corresponding to Corollary 1.3 is the following,

Corollary 1.6. If $G$ is any compact group of weight $a \geq \omega^{+}$, then there exists a compact group $G^{\prime}$, Haar homeomorphic to $G$ and a quotient $G^{\prime} / F$ of $G^{\prime}$ of weight $w\left(G^{\prime} / F\right)=a$, which is topologically isomorphic to a direct product of compact metric (Lie) groups.

Using Theorem 1.4 we can strengthen Sapirovskii's result itself. 
Corollary 1.7. Let $G$ be any compact group of weight $a \geq \omega^{+}$. Then there exists a continuous onto map $f: G \rightarrow[0,1]^{a}$ such that $f\left(\lambda_{G}\right)=\lambda$, where $\lambda_{G}$ is the Haar measure on $G$ and $\lambda$ the usual Lebesgue product measure on the cube $[0,1]^{a}$.

Proof. It is well known that if $X$ is a compact (metric) space and $\mu$ an atomless Radon probability measure on $X$, then there is a continuous onto map $g: X \rightarrow$ $[0,1]$ such that $f(\mu)=\lambda$ (see $[D]$, p. 211, ex. 7). Our assertion is now an easy consequence of this result together with Theorem 1.4 (cf. also the proof of Prop. 2.10 of section 2 ).

We now proceed to some applications both of Theorems 1.1 and 1.4.

Proposition 1.8. Let $G$ be a compact group with $w(G)=a \geq \omega^{+}$. Then there exist two cartesian products of compact metric spaces $X=\prod_{\xi<a} X_{\xi}, Y=\prod_{\xi<a} Y_{\xi}$ and two continuous (open) surjections $X \stackrel{p}{\rightarrow} G \stackrel{q}{\rightarrow} Y$ each with the property that maps compact $G_{\delta}$ sets to compact $G_{\delta}$ sets.

Proof. Combine Theorems 1.1 and 1.4 together with Lemma 1.6 of [J], according to which every continuous open surjection between compact spaces maps compact $G_{\delta}$ sets to compact $G_{\delta}$ sets.

Let $X$ be a compact space. Following Choksi and Fremlin (see [C-F]) we define $J(X)$ to be the least Maharam type of any completion regular measure on $X$. In the same paper it was proved that for every uncountable product of compact metric spaces $X=\prod_{\xi<a} X_{\xi}$ with $\left|X_{\xi}\right| \geq 2$ for all $\xi<a$, we have $J(X)=J\left(\{0,1\}^{a}\right.$ ) (see Prop. 1 of $[\mathrm{C}-\mathrm{F}])$. A recent result of the first named author asserts that the same is true for each compact group, namely the following

Theorem 1.9. (see $\left[\mathrm{G}_{1}\right]$, Th. 5.8). If $G$ is any compact group with $w(G)=a \geq$ $\omega^{+}$, then $J(G)=J\left(\{0,1\}^{a}\right)$.

Since there is a gap in the proof of that theorem given in $\left[\mathrm{G}_{1}\right]$ we are going to give (using our methods) a simple proof of this: Indeed, let $p, q$ be the continuous open surjections of Proposition 1.7. Then it follows from Prop. 1 of $[\mathrm{C}-\mathrm{F}]$ that $J(X)=J(Y)=J\left(\{0,1\}^{a}\right)$. Now if we apply Lemma 1 and Corollary in [C-F] (which together imply that, for a continuous surjection $f: X_{1} \rightarrow X_{2}$ between compact spaces $X_{1}, X_{2}$ that maps compact $G_{\delta}$ sets to compact $G_{\delta}$ sets we have $\left.J\left(X_{2}\right) \leq J\left(X_{1}\right)\right)$ we immediately get the conclusion.

Note. We notice that Theorem 1.9 (in combination with the other results of $\left[\mathrm{G}_{1}\right]$ ), allows the extension of the results of Choksi and Fremlin [C-F] (concerning products of compact metric spaces) to the field of compact groups (see also the related comments in $\left.\left[\mathrm{G}_{1}\right]\right)$.

A well known theorem of A. and C. Ionescu Tulcea [I-T] says that the Haar measure on every locally compact group admits a (left) invariant lifting. As far as we know there is no simplification of the deep and elaborate proof of this theorem, and there is no shorter proof of the existence of just a strong (not necessarily invariant) lifting for the (normalized) Haar measure on a compact group. In the sequel we shall give a rather simple proof of this last result as an application of Theorem 1.1.

Theorem 1.10. ([I-T]) The Haar measure on a compact group $G$ admits a strong lifting. 
Proof. It follows from Corollary 1.3 that $G$ is Haar homeoomorphic to a quotient group of a direct product of compact metric groups. So it is enough to prove the theorem for a group of the form $G_{1} / H$, where $G_{1}$ is topologically isomorphic to a direct product of compact metric groups (and $H$ a closed normal subgroup of $\left.G_{1}\right)$. Denote by $\lambda, \lambda_{H}, \mu$ the Haar measure on $G_{1}, H, G^{\prime}=G_{1} / H$ respectively, $\pi: G_{1} \rightarrow G^{\prime}$ the canonical map, and let $\rho$ be a strong lifting for $\lambda$ (see Th. 5, Ch. VIII of [I-T]). It follows from [I-T] that it is enough to find a linear strong lifting for $\mu$. We define for each $\dot{x} \in G^{\prime}$ a probability measure $\lambda_{\dot{x}}$ on $G_{1}$ by the rule,

$$
\lambda_{\dot{x}}(h)=\int_{H} h(x \cdot s) d_{\lambda_{H}}(s), \text { for every continuous function } h: G_{1} \rightarrow \mathbb{R} .
$$

Since the family $\left\{\lambda_{\dot{x}}: \dot{x} \in G^{\prime}\right\}$ is $\mu$-adequate and $\lambda=\int \lambda_{\dot{x}} d \mu(\dot{x})$ (see [I-T] and [B]), it follows that for every $g \in M_{\mu}^{\infty}$ (= the space of $\mu$-measurable bounded functions on $G^{\prime}$ ) the map,

$$
\ell_{g}: \dot{x} \in G^{\prime} \rightarrow \ell_{g}(\dot{x})=\lambda_{\dot{x}}(\rho(g \circ \pi)),
$$

is $\mu$-measurable (and bounded). Then it is easy to see that the map $\ell: M_{\mu}^{\infty} \rightarrow$ $M_{\mu}^{\infty}: g \rightarrow \ell_{g}$ defines a strong linear lifting for the measure $\mu$.

Note. The method of the construction of the strong lifting for the Haar measure on the quotient group $G_{1} / H$ is based on ideas of Kupka (see the "projection theorem" of $[\mathrm{Ku}]$ ).

The problem of whether the Radon product measure of two Radon completion regular Radon measures is again completion regular, is a central problem in Topological Measure Theory. If we restrict ourselves to (compact) dyadic spaces the answer to this problem is positive (see $[\mathrm{F}-\mathrm{G}]$ and the references given there); the proof of this fact does not seem to become simpler in the special case of compact groups. But as we shall see it is an easy consequence of Theorem 1.1.

Theorem 1.11. Let $G_{1}, G_{2}$ be compact groups and $\mu_{1}, \mu_{2}$ completion regular Radon measures on $G_{1}, G_{2}$ respectively. Then the Radon product measure $\mu_{1} \otimes \mu_{2}$ is completion regular on the group $G_{1} \times G_{2}$.

Proof. It clearly follows from Remark 1.2. (2) that $\mu_{1} \otimes \mu_{2}$ is a continuous open image of a product measure $\mu_{1}^{\prime} \otimes \mu_{2}^{\prime}$, where each $\mu_{i}^{\prime}, i=1,2$, is completion regular on a direct product of compact metric groups. Since the measure $\mu_{1}^{\prime} \otimes \mu_{2}^{\prime}$ is completion regular (see $[\mathrm{Gr}]$ ), it follows from Lemma 1 of [C-F] that $\mu_{1} \otimes \mu_{2}$ is also completion regular (cf. also the proof of Proposition 1.8 and of Theorem 1.9).

2 .

As we already mentioned in the first section of the present paper, the Haar measure $\lambda_{G}$ on a compact group $G$ is Baire isomorphic to a product measure $\mu$ on a product $X=\prod_{\xi<a} X_{\xi}(a=w(G))$ of compact metric spaces (see Th. 2.3 of $\left[\mathrm{G}_{2}\right]$ and also the proof of Case (2) of Theorem 1.1). The aim of this section is to prove a stronger result, in fact that the Baire isomorphism between $G$ and $X$ can be chosen to be a Jordan isomorphism (a concept that will be defined hereunder); this particularly implies that the Jordan algebras $J_{\lambda_{G}}, J_{\mu}$ of $\lambda_{G}$ and $\mu$ respectively are isomorphic. The proof of this result is a refinement of the proof of the original result given in $\left[\mathrm{G}_{2}\right]$ (Th. 2.3).

Definition 2.1. Let $X$ be a compact space and $\mu \in P(X)$. 
(i) A subset $\mathcal{B}$ of $J_{\mu}(X)$ (=the algebra of $\mu$-Jordan measurable sets) is said to be a set of generators for $J_{\mu}(X)$ if for every $A \in J_{\mu}(X)$ and each $\varepsilon>0$ there exist $B, \Gamma \in \mathcal{B}$ such that $B \subseteq A \subseteq \Gamma$ and $\mu(\Gamma \backslash B) \leq \varepsilon$.

(ii) Assume that $\mu$ is strictly positive. A subset $\mathcal{B}$ of $J_{\mu}$ (=the Jordan algebra of $\mu$ ) is said to be a set of generators for $J_{\mu}$ if for every $A \in J_{\mu}(X)$ and each $\varepsilon>0$ there exists $B, \Gamma \in J_{\mu}(X)$ with $\widehat{B}, \widehat{\Gamma} \in \mathcal{B}$, such that $B \subseteq A \subseteq \Gamma$ and $\mu(\Gamma \backslash B) \leq \varepsilon$.

(If $A, B$ are $\mu$-measurable sets we write $A \subseteq B$, if $\mu(A \backslash B)=0$ and $A \sim B$ if $A \subseteq B$ and $B \subseteq A$.)

It is clear that if $\mathcal{B} \subseteq J_{\mu}(X)$ is a set of generators for $J_{\mu}(X)$ (and $\mu$ is strictly positive) then the set $\widehat{\mathcal{B}}=\{\widehat{B} \in \mathcal{B}\}$ is a set of generators for $J_{\mu}$.

Remark 2.2. Let $\mu$ be a strictly positive (Radon probability) measure on the compact space $X$, and also let $A \subseteq X$ with the property that for every $\varepsilon>0$ there exist $B, \Gamma \in J_{\mu}(X)$ such that $B \subseteq A \subseteq \Gamma$ and $\mu(\Gamma \backslash B) \leq \varepsilon$. Then it is easy to show by using Theorem (C) (of the preliminaries) that there exists $A^{\prime} \in J_{\mu}(X)$ with $A \sim A^{\prime}$.

Proposition 2.3. Let $X_{1}, X_{2}$ be compact spaces, $\mu_{1} \in P\left(X_{1}\right), \mu_{2} \in P\left(X_{2}\right)$ strictly positive measures and $\mathcal{B}_{1}, \mathcal{B}_{2}$ Boolean subalgebras of $J_{\mu_{1}}, J_{\mu_{2}}$ respectively, which are sets of generators for $J_{\mu_{1}}, J_{\mu_{2}}$. If $\mathcal{B}_{1}$ and $\mathcal{B}_{2}$ are isomorphic then we have:

(i) $J_{\mu_{1}}$ and $J_{\mu_{2}}$ are isomorphic, and (hence)

(ii) the measure algebras $\mathcal{B}_{\mu_{1}}$ and $\mathcal{B}_{\mu_{2}}$ are isomorphic.

Proof. It easily follows using the definitions and standard results.

Proposition 2.4. Let $X$ be a compact space, $\mu \in P(X)$ and $\mathcal{B}$ be a basis for the topology of $X$ consisting of open $\mu$-Jordan measurable sets that is closed for finite unions. Then $\mathcal{B}$ is a set of generators for the algebra $J_{\mu}(X)$.

Proof. Let $A \in J_{\mu}(X)$ and $\varepsilon>0$. It follows from the inner regularily of $X$ that there is a compact set $K \subseteq A^{0}$ such that

$$
\mu\left(A^{0} \backslash K\right) \leq \varepsilon / 2 .
$$

Since $\mathcal{B}$ is a basis for the topology of $X$ closed for finite unions and $K$ is compact, there is $U \in \mathcal{B}$ with $K \subseteq U \subseteq A^{0}$; hence (since $\mu(A)=\mu\left(A^{0}\right)$ ) we get that

$$
\mu(A \backslash U) \leq \varepsilon / 2 .
$$

In a similar way we can find $V \in \mathcal{B}$ with $A \subseteq V$ and such that,

$$
\mu(V \backslash A) \leq \varepsilon / 2 \text {. }
$$

It follows from (1), (2) and (3) that $U \subseteq A \subseteq V, U, V \in \mathcal{B}$ and $\mu(V \backslash U) \leq \varepsilon$.

Remark 2.5. (1) If $X$ is a compact space and $\mu \in P(X)$, then it follows from the above proposition (and from the preliminaries) that the class of open (resp. open and Baire) $\mu$-Jordan measurable sets is a set of generators for $J_{\mu}(X)$.

(2) Let $\left\{X_{\xi}: \xi<a\right\}$ be a family of compact spaces and also let $\mu_{\xi} \in P\left(X_{\xi}\right)$ for $\xi<a$. We set $X=\prod_{\xi<a} X_{\xi}$ and $\mu=\bigotimes_{\xi<a} \mu_{\xi}$ for the Radon product measure on $X$. We consider the class $\mathcal{B}^{\prime}$ of basic open sets $U$ in $X$ of the form $U=$ 
$U_{\xi_{1}} \times \ldots \times U_{\xi_{n}} \times \prod_{\substack{\xi<a \\ \xi \neq \xi_{i}}} X_{\xi}, \xi_{1}<\ldots<\xi_{n}<a$, where each $U_{\xi_{i}}$ is open Baire and $\mu_{\xi_{i}}-$ Jordan measurable in the space $X_{\xi_{i}}, 1 \leq i \leq n$. Then it is easily verified that $\mathcal{B}^{\prime}$ is a basis for the topology of $X$ consisting of open Baire and $\mu$-Jordan measurable sets and hence, from the above proposition, the set $\mathcal{B}$ of finite unions of members of $\mathcal{B}^{\prime}$ is a set of generators for $J_{\mu}(X)$.

We now introduce the concept of Jordan measurability and examine its elementary properties; we also give some examples.

Definition 2.6. Let $X, Y$ be compact spaces and $\mu \in P(X), \nu \in P(Y)$.

(i) A measure preserving transformation $f: X \rightarrow Y$ (i.e., a measurable mapping $f: X \rightarrow Y$ such that $f(\mu)=\nu)$ is said to be Jordan measurable if for every $\nu$-Jordan measurable set $A \subseteq Y$ there is a $\mu$-Jordan measurable set $B \subseteq X$ such that $\mu\left(f^{-1}(A)+B\right)=0$ (where + denotes the symetric difference).

(ii) An invertible measure preserving transformation $f: X \rightarrow Y$ is said to be a Jordan isomorphism if both of the mappings $f$ and $f^{-1}$ are Jordan measurable. We say, in that case, that the measurable spaces $(X, \mu)$ and $(Y, \nu)$ are Jordan isomorphic.

We also admit isomorphisms $\bmod (0)$, i.e. transformations $f$ that became oneto-one and onto after discarding sets of measure zero from $X$ and $Y$.

Examples 2.7. (1) If $f: X \rightarrow Y$ is a continuous onto map such that $f(\mu)=\nu$, then clearly $f$ is Jordan measurable.

(2) If $f: X \rightarrow Y$ is a homeomorphism such that $f(\mu)=\nu$, then trivially $f$ is a Jordan isomorphism.

(3) A generalization of the second example is of interest for our purposes: Let $\mu, \nu$ be strictly positive (Radon probability) measures on $X$ and $Y$ respectively. Suppose that there exist measurable sets $A \subseteq X, B \subseteq Y$ with $\mu(A)=\nu(B)=1$ and a homeomorphism $f$ from $A$ onto $B$ such that $f(\mu)=\nu$. Then it follows from Theorem $(\mathrm{C})$ (of the preliminaries) that $f$ is a Jordan isomorphism (an isomorphism $\bmod (0))$ between $X$ and $Y$. For instance, let $I=[0,1]$ be the unit interval endowed with the Lebesgue measure $\lambda$, also let $X$ be any compact metric space and $\mu$ an (strictly positive) atomless measure on $X$. Then it is well known that there exist measurable sets $A \subseteq X, B \subseteq[0,1]$ with $\mu(A)=\lambda(B)=1$ (in particular $B$ can be chosen so that its complement is a countable set) and a homeomorphism $f$ from $A$ onto $B$ such that $f(\mu)=\lambda$ (see $[\mathrm{D}]$, p. 211, ex. $7(\mathrm{c})$ ). It follows that $(X, \mu)$ is Jordan isomorphic to $(I, \lambda)$.

The following proposition has a routine proof.

Proposition 2.8. Let $X, Y$ be compact spaces, $\mu \in P(X), \nu \in P(Y)$ strictly positive measures and $f: X \rightarrow Y$ a measure preserving (resp. an invertible measure preserving) transformation. If $f$ is Jordan measurable (resp. a Jordan isomorphism) then the mapping,

$$
F: \widehat{A} \in J_{\nu} \rightarrow F(\widehat{A})=f(A) \in J_{\mu}
$$

is an isomorphic embedding of $J_{\nu}$ into $J_{\mu}$ (resp. an isomorphism between $J_{\nu}$ and $\left.J_{\mu}\right)$. 
Remark 2.9. Let $X, Y$ be compact spaces and, $\mu \in P(X), \nu \in P(Y)$ strictly positive measures. If the Jordan algebras $J_{\mu}$ and $J_{\nu}$ are isomorphic, then the Stone representation spaces $X\left(J_{\mu}\right), Y\left(J_{\nu}\right)$ of $J_{\mu}, J_{\nu}$ respectively are homeomorphic by a homeomorphism $f$ such that $f(\bar{\mu})=\bar{\nu}$. This is an immediate consequence of Stone's representation theorem and of the definition of the measure $\bar{\mu}$ on $X\left(J_{\mu}\right)$ (see the preliminaries).

The following result asserts that the "Jordan type" of any product measure on an infinite product of compact metric spaces, only depends on the number of the spaces.

Proposition 2.10. Let $\left\{X_{\xi}: \xi<a\right\}, a \geq \omega$, be a family of compact metric spaces each having at least two points, and also let $\mu_{\xi} \in P\left(X_{\xi}\right)$ be a strictly positive measure, for $\xi<a$. We set $X=\prod_{\xi<a} X_{\xi}$ and $\mu=\bigotimes_{\xi<a} \mu_{\xi}$. Then the Jordan algebra $J_{\mu}$ is isomorphic to the Jordan algebra $J_{\nu}$ of the generalized Cantor space $Y=\{0,1\}^{a}$ endowed with the usual product (normalized Haar) measure $\nu$.

Proof. It is clear that the product measure $\mu$ is strictly positive and (since $a \geq \omega$ ) atomless. We divide the proof into two parts:

(I) We first assume that $a=\omega$. Then clearly the space $X$ is metrizable and since $\mu$ is strictly positive and atomless it follows from the classical result stated in Examples $2.7(3)$ that $(X, \mu)$ is Jordan isomorphic to $(I, \lambda)$ (the unit interval $I$ endowed with Lebesgue measure $\lambda$ ). Proposition 2.8 finishes the proof of this case.

It follows in particular from the previous remarks, that if $X$ is a given compact metric space and $\mu$ a strictly positive atomless (Radon probability) measure on $X$, then its Jordan algebra $J_{\mu}$ is isomorphic to the Jordan algebra $J_{\nu}$ of the Cantor space $\{0,1\}^{\omega}$ endowed with the usual product measure $\nu$. We conclude from this observation the existence of a stochastically independent sequence $\left\{V_{n}: n=1,2, \ldots\right\}$ of $\mu$-Jordan measurable sets with $\mu\left(V_{n}\right)=1 / 2$ for $n=1,2, \ldots$, such that the Boolean algebra generated by the sequence $\left\{\widehat{V}_{n}: n=1,2, \ldots\right\}$ is a set of generators for $J_{\nu}$.

(II) We assume that $a \geq \omega^{+}$. We notice that we can assume without loss of generality that each $\mu_{\xi}$ is atomless. (For if not, then if we consider a partition of the cardinal $a$ into $a$-many countably infinite sets $\left\{I_{\xi}: \xi<a\right\}$ and set $Z_{\xi}=\prod_{\eta \in I_{\xi}} X_{\eta}$, $\nu_{\xi}=\bigotimes_{\eta \in I_{\xi}} \mu_{n}$, then every $Z_{\xi}$ is compact metric and each $\nu_{\xi}$ is atomless on $Z_{\xi}$; finally let $Z=\prod_{\xi<a} Z_{\xi}$ and $\mu=\bigotimes_{\xi<a} \mu_{\xi}$. It is clear that $(X, \mu) \cong(Z, \nu)$, hence we can work with the measurable space $(Z, \nu))$. It follows from Proposition 2.3, that it is enough to find a stochasticaly independent family $\mathcal{F}=\left\{V_{\xi}: \xi<a\right\}$ of $\mu^{-}$ Jordan measurable sets, with $\mu\left(V_{\xi}\right)=1 / 2$ for $\xi<a$, such that the Boolean algebra generated by this family is a set of generators for $J_{\mu}$. We consider, for each $\xi<a$, a stochastically independent sequence $\left\{U_{\xi}^{n}: n<\omega\right\}$ of $\mu_{\xi}$-Jordan measurable sets with $\mu_{\xi}\left(U_{\xi}^{n}\right)=1 / 2$ for $n=1,2, \ldots$, such that the Boolean algebra derived from this sequence is a set of generators for $J_{\mu_{\xi}}$ (this follows from case (I) above). We 
set

$$
\mathcal{F}=\bigcup_{\xi<a}\left\{U_{\xi}^{n} \times \prod_{\substack{\zeta<a \\ \zeta \neq \xi}} X_{\zeta}: n=1,2, \ldots\right\} ;
$$

then it is easily verified using Remark $2.5(2)$, that $\mathcal{F}$ has the desired properties.

In order to prove the principal result of this section we shall need both of the following lemmas. The first one refines Lemma 2.1 of $\left[\mathrm{G}_{2}\right]$ and the second is (exactly the same with) Lemma 2.2 of $\left[\mathrm{G}_{2}\right]$ (cf. also Claim (II) in the proof of Theorem 1.1 of section 1).

Lemma 2.11. Let $G$ be an infinite compact group and $H$ a closed normal subgroup of $G$. If $H$ is Lie, then there is a Baire set $S$ in $G$ and an open set $U$ in $G / H$ of full Haar measure (i.e. $\lambda_{G / H}(U)=1$ ) such that:

(i) $G=S \cdot H$;

(ii) the mapping $\left.\pi\right|_{S}$ is a Baire isomorphism from $S$ onto $G / H$; in particular the set $\pi^{-1}(\{\dot{x}) \cap S$ is a single point $\{r(\dot{x})\}$ for each $\dot{x} \in G / H$ (as usual $\pi: G \rightarrow G / H: x \rightarrow \dot{x}$ is the canonical map);

(iii) the mapping $\left.\pi\right|_{r(U)}$ is a homeomorphism from $r(U)$ onto $U$ and (hence)

(iv) the bijection $q: G / H \times H \rightarrow G$, defined by the rule $q(\dot{x}, t)=r(\dot{x}) \cdot t$, is a Baire isomorphism such that:

(a) $q\left(\lambda_{G / H} \otimes \lambda_{H}\right)=\lambda_{G}$,

(b) the mapping $\left.q\right|_{U \times H}$ is a homeomorphism from $U \times H$ onto $r(U) \cdot H$ and (thus)

(c) $q$ is a Jordan isomorphism between $G / H \times H$ and $G$.

Proof. By a well known result of Gleason, there exists a compact set $F \subseteq G$ such that $\left.\pi\right|_{F}$ is one-to-one and the set $\pi(F)=V$ is a neighbourhood of the identity $e$ in $G / H$ (see Th. 1 in section 5.4 of [Mon-Zip]). Since each point of $G / H$ has a basis of neigbourhoods consisting of compact (or open) Baire $\lambda_{G / H^{-}}$Jordan measurable sets (see the preliminaries and Remark 2.5) we may assume that $V$ is compact Baire and $\lambda_{G / H^{-}}$Jordan measurable. We set $Q=F \cdot H$; therefore $Q=\pi^{-1}(\pi(F))=\pi^{-1}(V)$ is a compact Baire $\lambda_{G}$-Jordan measurable neighbourhood of the identity in $G$ and also $F$ is a Baire set in $G$. Let $\left\{Q_{0}=Q, Q_{1}=x_{1} \cdot Q, \ldots, Q_{N}=x_{N} \cdot Q\right\}$ be any finite cover of $G$. Since clearly $\left\{V=\pi(F), V_{1}=\pi\left(x_{1}\right) \cdot V, \ldots, V_{n}=\pi\left(x_{N}\right) \cdot V\right\}$ is a finite cover of $G / H$ consisting of compact Baire $\lambda_{G / H^{-}}$Jordan measurable sets, we can easily construct a finite cover $\left\{S^{1}, \ldots, S^{M}\right\}$ of $G / H$ of Baire $\lambda_{G / H^{-}}$Jordan measurable pairwise disjoint (non-empty) sets such that,

for each $m \in\{1,2, \ldots, M\}=I_{M}$ there is some $k \in\{1,2, \ldots, N\}=I_{N}$

$$
\text { with } S^{m} \subseteq \pi\left(x_{k} F\right)=V_{k} .
$$

For each $m \in I_{M}$, set $i_{m}=\min \left\{k \in I_{N}: S^{m} \subseteq V_{k}\right\}, S_{m}=\pi^{-1}\left(S^{m}\right) \cap\left(x_{i_{m}} F\right)$ and $S=\bigcup_{m=1}^{m} S_{m}$. It is clear that $\left\{S_{m}: m \in I_{M}\right\}$ is a family of pairwise disjoint Baire sets in $G$ such that for each $m \in I_{M}$ and each $\dot{x} \in S^{m}, \pi^{-1}(\{\dot{x}\}) \cap S_{m}$ is a single point, and also that the restriction of $\pi$ on $S_{m}$ is a homeomorphism from $S_{m}$ onto $S^{m}$. So the Baire set $S$ clearly satisfies assertions (i) and (ii) of the lemma. 
Let $r: G / H \rightarrow S$ be the inverse of the mapping $\left.\pi\right|_{S}$, so $r(\dot{x})=\pi^{-1}(\{\dot{x}\}) \cap S$ for $\dot{x} \in G / H$. We set $U=\bigcup_{m \in I_{M}} \operatorname{Int}\left(S^{m}\right)$; since each $S^{m}$ is $\lambda_{G / H^{-}}$Jordan measurable, the set $U$ is an open set of measure $\lambda_{G / H}(U)=1$. It is obvious from the above that each point of $U$ is a point of continuity of the mapping $r$. Since $\left.\pi\right|_{r(U)}$ is obviously continuous on $r(U)$, we get (iii). It follows from our construction that the mapping $q$ is a Baire isomorphism between the topological spaces $G / H \times H$ and $G$ that satisfies (a) (see also Remark 5.1 of $\left[\mathrm{G}_{1}\right]$ and the preliminaries). Condition (b) is an easy consequence of (iii) and the definition of $q$. Condition (c) is an immediate consequence of (a) and (b).

The proof of the lemma is complete.

The main tool for the proof of the following lemma is the classical fact (already mentioned in the proof of Theorem 1.4) that every neighbourhood $U$ of the identity $e$ of a compact group $G$ contains a closed normal subgroup $H$ such that the group $G / H$ is Lie.

Lemma 2.12. Let $G$ be a compact group with $w(G)=a \geq \omega$. Then there exists a family $\left(H_{\xi}\right)_{\xi<a}$ of closed normal subgroups of $G$ with $\bigcap_{\xi<a} H_{\xi}=\{e\}$ and $H_{0}=G$ such that

(i) $H_{\xi} \subseteq H_{\zeta}$ for $\zeta<\xi<a$,

(ii) $H_{\xi} / H_{\xi+1}$ is Lie, for every $\xi<a$, and

(iii) $H_{\xi}=\bigcap_{\zeta<\xi} H_{\xi}$, for every limit ordinal $\xi<a$.

Proof. We refer to $\left[\mathrm{G}_{2}\right]$ (Lemma 2.2). We also notice that the proof of this Lemma is similar to the elementary proof of Claim (II) of Theorem 1.1 of section 1 .

Theorem 2.13. Let $G$ be a compact group of weight $a \geq \omega$. Then there exists a family $\left(G_{\xi}\right)_{\xi<a}$ of compact Lie groups (each $G_{\xi}$ with at least two points if a $\geq$ $\left.\omega^{+}\right)$and a Baire isomorphism $q_{G}$ from $\prod_{\xi<a} G_{\xi}$ onto $G$ such that $q_{G}\left(\bigotimes_{\xi<a} \lambda_{\xi}\right)=$ $\lambda_{G}$, where $\lambda_{\xi}, \lambda_{G}$ denote the Haar measure on $G_{\xi}$ and $G$ respectively, which is furthermore a Jordan isomorphism.

Proof. Set $G_{0}=G / H_{1}$ and $G_{\xi}=H_{\xi} / H_{\xi+1}$ for $\xi>0$, where $\left(H_{\xi}\right)_{\xi<a}$ is the family of subgroups of $G$ descibed in Lemma 2.12. We proceed by induction on $\xi<a$, using Lemmas 2.11, 2.12, Remarks 2.2, 2.5(2) and Proposition 2.3, in order to prove the existence of the desired isomorphism between the groups $G$ and $\prod_{\xi<a} G_{\xi}$. Since this proof is similar to the proof of Th. 2.3 in $\left[\mathrm{G}_{2}\right]$, we refer the reader for the details there (cf. also the proof of Claim (II) of Theorem 1.1).

An immediate consequence of the above theorem together with Propositions 2.8 and 2.10 is the following.

Corollary 2.14. If $G$ is any compact group with $w(G)=a \geq \omega$ and $\lambda_{G}$ the Haar measure on $G$, then the Jordan algebra $J_{\lambda_{G}}$ is isomorphic to the Jordan algebra $J_{\nu}$ of the generalized Cantor space $\{0,1\}^{a}$ endowed with the usual product measure $\nu$.

If $X$ is a compact space and $\mu$ a strictly positive Radon probability measure on $X$, then the space $R(X, \mu)$ of $\mu$-Riemann integrable functions on $\mathbf{X}$ is defined as 
the subspace of $L^{\infty}(\mu)$ consting of those classes of bounded $\mu$-measurable functions which contain a $\mu$-Riemann integrable function. It easily follows from Theorem (C) that $R(X, \mu)$ is a closed subspace (in fact a subalgebra) of $L^{\infty}(\mu)$. It is also proved by using standard results, that $R(X, \mu)$ is isometric to the Banach space $C\left(X\left(J_{\mu}\right)\right)$. Now it clearly follows from Corollary 2.14 together with Remark 2.9 the following,

Corollary 2.15. If $G$ is any compact group with $w(G)=a \geq \omega$, then the space $R\left(G, \lambda_{G}\right)$ of $\lambda_{G}$-Riemann integrable functions on $G$ is isometric to the space $R\left(\{0,1\}^{a}, \nu\right)$ of $\mu$-Riemann integrable functions on $\{0,1\}^{a}$.

We notice that the above corollary should be compared with the following result of Pelczynski ([P], Th. 8.9): If $G$ is a compact group with $w(G)=a \geq \omega$, then the Banach spaces $C(G)$ and $C\left(\{0,1\}^{a}\right)$ are isomorphic.

Note. Theorem 2.13 should be compared with both the principal results of section 1. Theorems 1.1 and 1.4 together assert that the Haar measure on any compact group $G$ is enclosed between two product measures (each on some product of compact metric spaces) by means of two continuous and open surjections. Theorem 2.13 says that the Haar measure on $G$ is just isomorphic to a proper product measure (on some product of compact metric spaces) by a Baire and Jordan isomorphism. If $G$ is totally disconnected then from case (2) of Theorem 1.1 this isomorphism becomes a homeomorphism.

It is easy to verify that if two Radon probability measures have isomorphic Jordan algebras then they also have isomorphic measure algebras and hence the same Maharam type (see Proposition 2.3). The converse is not true as it follows from the examples which we shall describe.

Examples. (1) For every infinite cardinal $a$, there exist a compact space $S$ and two strictly positive Radon probability measures on $S$, homogeneous of Maharam type $a$, the Jordan algebras of which are not isomorphic. Indeed, let $X=\{0,1\}^{a}$ and $\lambda$ be the (normalized) Haar measure on $X$. We consider a strictly positive Radon probability measure $\mu$ on $X$, homogeneous of Maharam type $a$ but not equivalent to $\lambda$ (that is, $\lambda$ and $\mu$ do not have the same negligible sets) (cf. [I-T], ch. VII, Ex. $2)$. Let $(S, \nu)$ be the Stone space (hyperstonian) of the measure space $(X, \mu)$ and $\pi=\pi_{\mu}: S \rightarrow X$ the canonical map (see [I-T], ch. IX and [Di]). It is clear that there exists a Radon probability measure $\sigma$ on $S$, homogeneous of Maharam type $a$, such that $\pi(\sigma)=\lambda$; such a measure of course is not equivalent to $\nu$. We notice that we can assume that $\sigma$ is strictly positive (for if not, we could replace and work with the measure $\frac{1}{2} \nu+\frac{1}{2} \sigma$ ). The measures $\nu$ and $\sigma$ on $S$ have the desired properties, because $\nu$ is a normal measure [Di] (hence its Jordan algebra is identified with its measure algebra, which is order complete), but on the other hand $\sigma$ is not normal (since it is not equivalent to $\nu$ ).

(2) For still another example of this kind, we need the concept of countably determined measures: A Radon (probability) measure $\mu$ on a compact space $X$ is said to be countably determined (resp. uniformly regular) if there exists a sequence $\left(K_{n}\right)$ of compact (resp. compact $G_{\delta}$ ) subsets of $X$, such that for every open subset $U$ of $X$ and each $\varepsilon>0$ there exists $n \in \omega$ such that, $K_{n} \subseteq U$ and $\mu\left(U \backslash K_{n}\right) \leq \varepsilon$. (see references in $[\mathrm{M}]$; see also $[\mathrm{M}]$, Def. 1.1 and 1.5 (i)). It is easy to see that there exists a strictly positive, atomless, countably determined measure $\mu$ on the space $X=\{0,1\}^{c}$; such a measure $\mu$ is not uniformly regular, because the space $X$ admits no uniformly regular measure (see $[\mathrm{M}]$, Remark 2.18). It then follows from Theorem 1.7 of $[\mathrm{M}]$ that the Jordan algebra $J_{\mu}$ of $\mu$ is not isomorphic to the Jordan 
algebra $J_{\lambda}$ of the unit interval $I=[0,1]$ endowed with Lebesgue measure $\lambda$, despite the fact that the measures $\mu$ and $\lambda$ have the same (countable) Maharam type (see also Th. 1.9 and Remark 1.10 of $[\mathrm{M}])$.

As an illustration of our methods, we shall give a proof (in fact two proofs) of the well known result (the general case of which is due to Veech) concerning the existence of uniformly distributed sequences in compact groups (see $[\mathrm{V}]$, also [K-N] Coroll. 5.4, pp. 301-302 and p. 235). We recall that a sequence $\left(x_{n}\right)$ in a compact space $X$ is said to be $\mu$-uniformly distributed ( $\mu$-u.d.) in $X$ with respect to the measure $\mu \in P(X)$, if for all continuous functions $f: X \rightarrow \mathbb{R}$ we have,

$$
\lim _{N \rightarrow \infty} \frac{1}{N} \sum_{n=1}^{N} f\left(x_{n}\right)=\int_{X} f d \mu \quad \text { (see Def. 1.1, p. } 171 \text { of }[\mathrm{K}-\mathrm{N}] \text { ). }
$$

We notice that if $X$ is compact metric then, every $\mu \in P(X)$ admits a $\mu$-u.d. sequence $[\mathrm{K}-\mathrm{N}]$; the same holds true for each (compact) dyadic space $X$ with $w(X) \leq \omega^{+}\left[\mathrm{L}_{1}\right]$. We also refer the reader to $[\mathrm{M}]$ for further classes of compact spaces with the same property.

Lemma 2.16. Let $X$ be a compact space, $\mu \in P(X)$ a strictly positive measure and $\left(x_{n}\right)$ a sequence in the Stone space $X\left(J_{\mu}\right)$ of the Jordan algebra $J_{\mu}$. Then $\left(x_{n}\right)$ is $\bar{\mu}-u . d$. in $X\left(J_{\mu}\right)$ iff the sequence $\pi\left(x_{n}\right)$ is $\mu-u$.d. in $X$ (where $\pi: X\left(J_{\mu}\right) \rightarrow X$ is the canonical map from $X\left(J_{\mu}\right)$ onto $X$ and $\bar{\mu}$ the corresponding measure on $X\left(J_{\mu}\right)$ mentioned in the preliminaries).

Proof. It is an easy consequence of the properties of $\pi$ (given in the preliminaries) and of the definition of uniform distribution (cf. $\left[\mathrm{L}_{2}\right]$, ex. (5) and also the proof of Th. 2.9 in $[\mathrm{M}])$.

Theorem 2.17. (Veech $[\mathrm{V}]$ ) Let $G$ be a compact separable group. Then the Haar measure $\lambda$ on $G$ admits a u.d. sequence.

Proof. We shall need the following.

Claim. If $X=\prod_{\xi<c} X_{\xi}$ is a product of at most $c$ compact metric spaces (with $\left|X_{\xi}\right| \geq 2$ for all $\xi<c$ ), and $\mu_{\xi}$ is a (strictly positive) Radon probability measure on $X_{\xi}$ for all $\xi<c$, then the Radon product measure $\mu=\bigotimes_{\xi<c} \mu_{\xi}$ on $X$ admits a $\mu$-u.d. sequence.

Proof of the Claim. Since by Proposition 2.10 the Jordan algebra $J_{\mu}$ is isomorphic to the Jordan algebra of the usual Lebesgue product measure $\lambda$ on the qube $\Omega=$ $[0,1]^{c}$, it follows by Remark 2.9 and Lemma 2.16 that it is enough to show the existence of a $\lambda$-u.d. sequence in $\Omega$. So let $L$ be a subset of the real numbers of cardinality $c$ such that the set $L \cup\{1\}$ is linearly independent over the rationals (clearly every element of $L$ is an irrational number). By identifying the set $L$ with its cardinal $c$, we define a sequence $\left(x_{n}\right)$ in $\Omega$ in the following way: $x_{n}(\theta)=n \theta-[n \theta]$, for $n=1,2, \ldots$ and $\theta \in L$ (where $[x]$ denotes the integral part of the real number $x$ ). It then follows from standard results that the sequence $\left(x_{n}\right)$ is $\lambda$-u.d. in $\Omega$ (see [K-N], ch. 1, par. 6, pp. 47-50, and also [H-R], 26.19, 26.20, pp. 435-438).

Now we are ready to complete the proof of the theorem. We first notice that if the Haar measure $\lambda_{G}$ on a compact group $G$ admits a u.d. sequence then (since $\lambda_{G}$ is strictly positive) $G$ is necessarily separable and thus $w(G) \leq c$. We also notice that if $f: X \rightarrow Y$ is a continuous surjection between the compact spaces $X$ and 
$Y, \mu \in P(X)$, and $\left(x_{n}\right)$ is a $\mu$-u.d. sequence in $X$, then $y_{n}=f\left(x_{n}\right), n=1,2, \ldots$, is $f(\mu)$-u.d. in $Y$. So, our assertion follows immediately either from the Claim and Theorem 1.1, or from the Claim together with Corollary 2.14, Remark 2.9 and Lemma 2.16.

We conclude with the following questions:

(1) Let $G$ be a compact non-metrizable group. Does there exist a (compact) neighbourhood of the identity in $G$ which is homeomorphic to a (compact) neighbourhood of the identity in some direct product of compact metric groups?

If $G$ is totally disconnected the answer is of course positive. Of special interest are the cases of connected or abelian $G$.

(2) Does there exist a strictly positive Radon probability measure $\mu$ on the space $X=\{0,1\}^{a}, a \geq \omega^{+}$, homogeneous of Maharam type $a$ such that the Jordan algebra $J_{\mu}$ of $\mu$ is not isomorphic to the Jordan algebra $J_{\lambda}$ of the usual product measure $\lambda$ on $X$ ? Of special interest is the case $\omega^{+} \leq a \leq c$.

We notice that a similar question has been posed in $[\mathrm{M}]$.

\section{REFERENCES}

[B] N. Bourbaki, Integration, Ch. 8, Hermann, Paris, 1959-1967. MR 31:3539

[Ch] M.M. Choban, Baire isomorphisms and Baire topologies. Solution of a problem of Comfort, Soviet Math. Dokl., 30 (1984), 780-784. MR 87d:54029

[C-F] J.R. Choksi and D.H. Fremlin, Completion regular measures on product spaces, Math. Ann. 241 (1979), 113-128. MR 81m:28004

[Cl-Mol $\mathrm{Moan}_{1}$ Cleary and Sidney A. Morris, Locally dyadic topological groups, Bull. Austral. Math. Soc., 40 (1989), 417-419. MR 90m:22005

[Cl-Mo2] Joan Cleary and Sidney A. Morris, Compact groups and products of the unit interval, Math. Proc. Camb. Phil. Soc. 110 (1991), 293-297. MR 92k:22005

[Co] W.W. Comfort, Topological groups. In: K. Kunen, J.E. Vaughan (eds.) Handbook of Set-theoretic Topology, Ch. 24, pp. 1143-1263, Amsterdam: North-Holand 1984. MR 86g:22001

[Co-Ho-Re] W.W. Comfort, K.H. Hofmann and D. Remus, Topological Groups and Semigroups, Recent Progress in General Topology, M. Husek and J. Van Mill (eds.), Elsevier Sciences Publishers, 1992. CMP 93:15

[Co-Re] W.W. Comfort and Dieter Remus, Pseudocompact Refinements of Compact Group Topologies, Math. Z. 215 (1994), 337-346. MR 95f:54035

[D] J. Dieudonne, Treatise on Analysis, Vol II, Academic Press, New York, 1976. MR 58:26627

[Di] J. Dixmier, Sur certains espaces consideres par M.H. Stone, Sum. Bras. Math. 2 (1951), 151-181. MR 14:69e

[E] D.A. Edwards, On independent group characters, Bull. Amer. Math. Soc., 65 (1959), 352-354. MR 21:5692

[F-G] D.H. Fremlin and S. Grekas, Products of completion regular measures, Fund. Math. 147 (1995) N. 1, 27-37. MR 96e:28003

$\left[\mathrm{G}_{1}\right] \quad$ S. Grekas, Isomorphic measures on compact groups, Math. Proc. Camb. Phil. Soc., 112 (1992), 349-360 (and a corrigendum in Math. Proc. Camb. Phil. Soc., 1994).

$\left[\mathrm{G}_{2}\right] \quad \mathrm{S}$. Grekas, Structural properties of compact groups with measure theoretic applications, Israel J. Math. 87 (1994), 89-95. MR 95g:28025

$\left[\mathrm{G}_{3}\right]$ S. Grekas, Measure-theoretic problems in topological dynamics, J. d'Analyse Math. 65 (1995), 207-220. MR 96e:54003

[Gr] C. Gryllakis, Product of completion regular measures, Proc. Amer. Math. Soc. 103 (1988), 563-568. MR 89d:28010

[H-R] Edwin Hewitt and Kenneth A. Ross, Abstract Harmonic Analysis I, Spinger-Verlag 1963. MR 28: 158

[I-T] A. \& C. Ionescu Tulcea, Topics in the theory of lifting, Springer-Verlag, Berlin Heidelberg - New York 1969. MR 43:2185 
[J] R.A. Johnson, Disintegrating measures on compact group extensions, A. Wahr. Verw. Gebiete, 53 (1980), 271-281. MR 81k:28009

[K-N] L. Kuipers and H. Niederreiter, Uniform Distribution of Sequences, J. Wiley, New York 1974. MR 54:2612

[Kuz] V. Kuz'minov, On a hypothesis of P.S. Alexandroff in the theory of topological groups, Dokl. Akad. Nauk, SSSR, 125 (1959), 727-729. MR 21:3506

$[\mathrm{Ku}] \quad$ J. Kupka, Strong liftings with application to measurable cross sections in locally compact groups, Israel J. Math. Vol. 44, No 3 (1983), 243-261. MR 84g:28006

$\left[\mathrm{L}_{1}\right] \quad$ V. Losert, On the existence of uniformly distributed sequences in compact topological spaces I, Trans. Amer. Soc. Vol. 246 (1978), 463-471. MR 80f:10062a

$\left[\mathrm{L}_{2}\right] \quad$ V. Losert, On the existence of uniformly distributed sequences in compact topological spaces II, Mh. Math. 87 (1979), 247-260. MR 80f:10062b

$\left[\mathrm{L}_{3}\right] \quad$ V. Losert, Strong liftings for certain classes of compact spaces, Measure Theory Oberwolfach 1981, Lecture Notes in Math. 945, Springer Verlag, Berlin - Heildeberg - New York, 170-179. MR 84h:28010

[M] S. Mercourakis, Some remarks on countably determined measures and uniform distribution of sequences, Mh. Math., 121 (1996), 79-111. MR 97j:28029

[Mon-Zip] D. Montgomery and L. Zippin, Topological Transformation Groups, Interscience 1955. MR 17:383b

[Mos] Paul S. Mostert, Sections in principal fibre spaces, Duke Math. J. 23 (1956), 57-71. MR 17:771f

[P] A. Pelczynski, Linear extensions, linear averagings, and their applications to linear topological classification of spaces of continuous functions, Dissertationes Math. 58 (1968). MR 37:3335

[Pr] John F. Price, Lie Groups and Compact Groups, Cambridge University Press 1977. MR 56:8743

[V] W.A. Veech, Some questions of uniform distribution, Ann. of Math. (2) 94 (1971), 125-138. MR 44:4187

Department of Mathematics, University of Athens, Panepistemiopolis, 15784 Athens, Greece

E-mail address: sgrekas@eudoxos.dm.uoa.gr

E-mail address: smerkour@eudoxos.dm.uoa.gr 